\title{
Video Article \\ Translational Brain Mapping at the University of Rochester Medical Center: Preserving the Mind Through Personalized Brain Mapping
}

\author{
Bradford Z. Mahon ${ }^{1,2}$, Jeffrey A. Mead ${ }^{3}$, Benjamin L Chernoff ${ }^{2}$, Maxwell H. Sims ${ }^{5}$, Frank E. Garcea ${ }^{4}$, Emily Prentiss ${ }^{5}$, Raouf Belkhir ${ }^{2}$, Sam \\ J. Haber ${ }^{1}$, Sarah B. Gannon ${ }^{5}$, Steve Erickson ${ }^{5}$, Kelly A. Wright ${ }^{5}$, Michael Z. Schmidt ${ }^{5}$, Audrey Paulzak ${ }^{1}$, Vanessa C. Milano ${ }^{1}$, David A. Paul ${ }^{1}$, \\ Kenneth Foxx ${ }^{1}$, Madalina Tivarus ${ }^{7,8}$, Jacob W. Nadler ${ }^{6}$, Jacqueline M Behr ${ }^{1}$, Susan O. Smith ${ }^{1}$, Yan Michael Li ${ }^{1}$, Kevin Walter ${ }^{1}$, Webster H. Pilcher ${ }^{1}$ \\ ${ }^{1}$ Department of Neurosurgery, University of Rochester Medical Center \\ ${ }^{2}$ Department of Psychology, Carnegie Mellon University \\ ${ }^{3}$ Public Relations and Communications, University of Rochester Medical Center \\ ${ }^{4}$ MOSS Rehabilitation Research Institute, Cognitive Neuroscience \\ ${ }^{5}$ University of Rochester Medical Center \\ ${ }^{6}$ Department of Anesthesiology and Perioperative Medicine, University of Rochester Medical Center \\ ${ }^{7}$ Department of Imaging Sciences, University of Rochester Medical Center \\ ${ }^{8}$ Department of Neuroscience, University of Rochester Medical Center
}

Correspondence to: Bradford Z. Mahon at bmahon@andrew.cmu.edu

URL: https://www.jove.com/video/59592

DOI: doi:10.3791/59592

Keywords: Neuroscience, Issue 150, brain tumor, brain surgery, neurosurgery, brain mapping, translational brain mapping, fMRI, DTI, functional MRI, MRI, Diffusion Tensor Imaging, awake brain surgery, awake craniotomy, human brain map, direct electrical stimulation, epilepsy, glioma, glioblastoma, frontal aslant tract, SMA syndrome, motor cortex, cortical language centers, eloquent cortex

Date Published: 8/11/2019

Citation: Mahon, B.Z., Mead, J.A., Chernoff, B.L., Sims, M.H., Garcea, F.E., Prentiss, E., Belkhir, R., Haber, S.J., Gannon, S.B., Erickson, S., Wright, K.A., Schmidt, M.Z., Paulzak, A., Milano, V.C., Paul, D.A., Foxx, K., Tivarus, M., Nadler, J.W., Behr, J.M., Smith, S.O., Li, Y.M., Walter, K., Pilcher, W.H. Translational Brain Mapping at the University of Rochester Medical Center: Preserving the Mind Through Personalized Brain Mapping. J. Vis. Exp. (150), e59592, doi:10.3791/59592 (2019).

\section{Abstract}

The Translational Brain Mapping Program at the University of Rochester is an interdisciplinary effort that integrates cognitive science, neurophysiology, neuroanesthesia, and neurosurgery. Patients who have tumors or epileptogenic tissue in eloquent brain areas are studied preoperatively with functional and structural MRI, and intraoperatively with direct electrical stimulation mapping. Post-operative neural and cognitive outcome measures fuel basic science studies about the factors that mediate good versus poor outcome after surgery, and how brain mapping can be further optimized to ensure the best outcome for future patients. In this article, we describe the interdisciplinary workflow that allows our team to meet the synergistic goals of optimizing patient outcome and advancing scientific understanding of the human brain.

\section{Video Link}

The video component of this article can be found at https://www.jove.com/video/59592/

\section{Introduction}

Neurosurgical interventions to remove brain tumors or epileptogenic tissue adjacent to brain areas that support critical cognitive functions must balance the clinical objective of the surgery (remove as much tumor, or epileptogenic tissue as possible) against damage to healthy tissue that could cause neurologic deficits. In the context of brain tumor surgery, this balance is referred to as the onco-functional balance. On the 'onco' side of the balance, surgeons want to remove as much of the tumor as possible, as rates of 'gross total tumor resection' are linked to longer survival ${ }^{1,2}$. On the 'functional' side, removal of tumors can damage cortical and subcortical substrates of cognition; post-operative difficulties can involve language, action, vision, hearing, touch or movement, depending on the neural system(s) affected. The onco-functional balance is critically important because increased morbidity is associated with $i$ ) lower quality of life, ii) increased post-operative complications that can increase mortality (e.g., patients who can no longer move are at a higher risk of blood clots ${ }^{3,4}$ ). The tension inherent in the 'onco-functional' balance in the setting of brain tumor surgery translates as well to epilepsy surgery - there the balance is between the clinical objective of removing all tissue that is generating seizures, while not removing tissue that supports critical functions.

At a broad level, functional neuroanatomy is highly stereotyped from individual to individual. However, there can be a high degree of individual variability in the precise (i.e., $\mathrm{mm}$ to $\mathrm{mm}$ ) location of higher cortical functions. In addition, it is generally recognized that the presence of cortical or subcortical pathology can spur cortical reorganization, although the principles that drive such reorganization are poorly understood ${ }^{5}$. Neurosurgical interventions proceed millimeter by millimeter. It is thus critical to map each patient's brain, in detail and with sensitivity and precision, in order to understand which regions in that specific patient support which sensory, cognitive and motor functions ${ }^{6}$. 
The Program for Translational Brain Mapping at the University of Rochester has been engineered to meet the needs of personalized brain mapping in the setting of a high through-put practice spanning multiple academic surgeons. The synergistic goals of the Brain Mapping Program are to $i$ ) use the tools of cognitive neuroscience to advance personalized neuromedicine, in the form of patient-specific functional brain maps, and ii) use the clinical preparation of neurosurgical interventions to test mechanistic hypotheses about how the human brain functions.

\section{Protocol}

The activities shown in the video and described herein fall within a greater-than-minimal-risk IRB at the University of Rochester Medical Center.

\section{Recruitment}

1. Establish a high through-put program for pre-operative cognitive and MRI-based assessment to catch patients from all referring providers in a timely and efficient manner. Involve the administrative and clinical staff in the broader effort.

NOTE: A concrete step that has proved effective was the establishment of a group email list that is automatically sent by the attending surgeon (or someone on their support staff) when a new patient presents to clinic who may be a candidate for recruitment into the Brain Mapping Program.

\section{Pre-Operative MRI mapping}

1. Acquire MRI data on a 3T MRI scanner with a 64-channel head coil at the Center for Advanced Brain Imaging and Neurophysiology (formally known as the 'Rochester Center for Brain Imaging') at the University of Rochester Medical School. Use standard sequences for BOLD MRI and DTI allowing full brain imaging, as described in prior publications $7,8,9,10,11,12,13,14,15,16,17,18,19,20,21,22,23,24,25$.

2. Monitor fixation, and record respiration and heart rate collected during all fMRI for regression of noise confounds ${ }^{26,27}$. NOTE: Over the past 10 years, we have developed a library of functional MRI experiments to map language (spoken, auditory, single words, whole sentences), motor function (from intransitive finger, tongue and foot movements to high level transitive actions), music ability, mathematics and number knowledge, and basic sensory function (e.g., retinotopic mapping to map of low level visual processing ${ }^{11,14,24}$ ). All experiments, materials, and analysis scripts are available at www.openbrainproject.org.

\section{Neuropsychological testing}

1. Take care during all cognitive testing to ensure that patients are comfortable, ensured using an ergonomically optimized setup (Figure 1) and by building frequent breaks (every $8 \mathrm{~min}$ ) into the structure of all tests.

2. Have all low grade tumor patients complete the following tests 1 month before surgery, 1 month after surgery, and 6 months after surgery (tests 12 and 13 are completed only at the pre-operative and 6 months post-operative time points) $28,29,30,31,32$.

1. Spontaneous Speech (Cookie Theft Picture ${ }^{33}$, Cinderella Story ${ }^{34,35,36}$ ).

2. Category Fluency (actions, semantic categories, words starting with $F, A, S$ ).

3. Word Reading and Repetition (nouns, verbs, adjectives, non-words, matched on length and frequency).

4. Snodgrass Object Naming $\left(n=260^{37}\right)$.

5. Auditory Naming $\left(n=60^{38}\right)$.

6. High-Cloze Sentence Completion (30 min).

7. Birmingham Object Recognition Battery (BORB, including Length | Size | Orientation | Gap Matching | Overlapping Figures | Foreshortened Views | Object Reality Decision $\left.{ }^{39}\right)$.

8. Auditory Minimal Pair Discrimination (e.g., pa vs. da, ga vs. $t a^{31,40}$ ).

9. Sentence Picture Matching (including reversible passives ${ }^{40}$ ).

10. Color Naming and Farnsworth Munsell Hue Sorting ${ }^{41}$.

11. Cambridge Face Test ${ }^{30,42}$.

12. California Verbal Learning Test $\left({ }^{43}\right)$

13. Weshler IQ $\left({ }^{44,45,46}\right)$. The key measures to evaluate language outcome are tests $4-6$; characterizing broader abilities ensures impairments on naming tests are not due to general performance decline ${ }^{47}$.

NOTE: In the past, we have used a combination of software presentation platforms to control stimulus presentation and response recording during pre- and post-operative testing. We are currently designing a single plug-and-play platform to support all cognitive testing (pre, intra- and post-operative testing) as well as stimulus presentation and response recording during functional MRI (see below for description of StrongView ${ }^{\mathrm{TM}}$ ). StrongView, together with built-in neuropsychological tests, will be available for download (open license) at www.openbrainproject.org.

\section{Neuroanesthesia and ergonomics of intraoperative language mapping}

1. Use anesthetic techniques for awake craniotomies ${ }^{48,49,50}$; at the University of Rochester, awake craniotomies are typically performed using an asleep-awake-asleep approach.

2. Avoid premedications such as anticonvulsants and anxiolytics as they can impair cognitive function and contribute to emergence delirium.

3. Apply standard monitors (EKG, NIBP, pulse oximetry) and induce general anesthesia with intravenous fentanyl (0.5 mg/kg), lidocaine (1-1.5 $\mathrm{mg} / \mathrm{kg}$ ) and propofol (1-2 $\mathrm{mg} / \mathrm{kg})$.

4. Use a supraglottic airway for mechanical ventilation.

5. Position the patient laterally or semi-laterally with the head secured in a pinned frame; as described in the video, patient positioning depends on the location of the lesion and the planned craniotomy window, while also taking into consideration that types of cognitive testing the patient will be asked to perform once awake during the surgery. 
6. Apply analgesia at the pin and incision site ( $30 \mathrm{~mL}$ of $0.5 \%$ Lidocaine, $30 \mathrm{~mL}$ of $0.5 \%$ Sensorcaine plain, $6 \mathrm{~mL}$ of sodium bicarbonate). During this period, position the testing equipment (small monitor, video cameras, directional mics).

7. Determine the size of the craniotomy window by multiple factors, which vary in their weighting according to the results of pre-operative clinical mapping of the patient's brain, functional brain mapping studies, and the plan for intra-operative mapping. In the case described in the video, the attending surgeon (Dr. Pilcher) chose a large craniotomy in order to have full access to map positive language and motor sites in the dominant hemisphere.

8. At the beginning of the awake-phase, discontinue sedation (local analgesics are applied prior to incision)

9. Remove the supraglottic airway once the patient regains consciousness. There is no or minimal sedation during the awake phase.

10. Use electrocorticography (ECoG) to monitor after-discharges (subclinical epileptiform discharges induced by cortical stimulation) to ensure that DES levels are set at just below the after-discharge threshold. The DES mapping procedure initiates by finding the after-discharge threshold, and adjusting stimulation amplitude (in steps of .5 milliamp).

11. Adjust stimulation amplitude throughout the mapping session (2 to $15 \mathrm{~mA})$ at the discretion of the attending surgeon. Patients view stimuli on a monitor and can speak and move their forearms and hands.

\section{Procedures for acquiring research-grade data during intraoperative direct electrical stimulation mapping}

1. Run all intraoperative cognitive testing on a custom-built hardware/software system called 'StrongView', available at www.openbrainproject.org. The hardware footprint is self-contained on a small cart, and is outfitted with an independent backup battery power source, speakers, keyboard and touch display. The person charged with running the cognitive testing can start, stop and pause stimulus presentation, while continuously recording (audio and video) during the case.

2. Use an audio system on the cart such that a directional microphone that is trained on the patient's mouth, which feeds through a splitter. One channel coming out of the splitter goes through an amplifier and directly to a speaker. This allows surgeons and researchers to easily hear the patient's responses against the background noise of the operating room with zero perceptible delay (i.e., eliminating 'echo' effects). The second channel from the splitter goes to the PC on the mobile cart, where it is time-stamped, recorded and stored (these files are used for offline analysis). StrongView also has a separate (stand-alone) audio system that consists of a second directional microphone also trained on the patient, a directional microphone trained on the surgeons, and a 'noise' microphone in a corner of the operating room to sample room tone for subtraction from the main audio files. Those three audio channels feed to a MIDI, and to a second computer which records each channel separately. This second audio system provides redundancy should the primary system fail, all verbal responses of the patient will be available for offline analysis.

3. Attach a commercially available ether screen L-bracket to the operating room (OR) table using an OR table clamp. Attach articulating arms (e.g., Manfrotto 244 Variable Friction Magic Arms) to the ether screen L-bracket, and those articulating arms support the patient monitor, directional microphones, video camera trained on the patient's face, and an auxiliary monitor to allow a research team member or operating room nurse to easily see what the patient sees while interacting with the patient.

4. Run all necessary cables for the screens, microphones, and camera along the arm and protect by plastic tubing secured with Velcro NOTE: None of this equipment needs to be sterilized as it is (only ever) on the non-sterile side of the field (Figure 1). This manner of supporting stimulus presentation and response recording equipment provides maximal flexibility to take into account the differing ergonomics of cognitive testing according to patient positioning which varies case by case, yet provides a reliable and stable platform on which to attach equipment. Also, and importantly, because all monitors, microphones and cameras are attached to the OR table via single device (ether screen L-bracket), if the positioning of the table is adjusted during the case this does not affect the testing setup. (Note that the setup shown in Figure 1 is from an earlier generation setup in which a floor-mounted stand supported the patient screen, microphone and video camera; that floor mounted stand has been replaced since 2018 with the ether screen L-bracket). Also, and importantly for patient safety, the entire setup for cognitive testing can be broken down in less than 20 seconds during the case should an emergent situation present itself that mandates full and unobstructed access to the patient (e.g., to the patient's airway).

5. The heart of StrongView is a flexible software system for i) presenting stimuli (visual, auditory) to patients and recording patient responses (verbal, button response, video), ii) temporally registering all experimentally-relevant events and measures (stimulus on, ECoG, contact with brain of direct electrical stimulator probe, patient responses); iii) and communication with cranial navigation systems to obtain the 3 dimensional coordinate for each application of direct electrical stimulation. StrongView allows on-line re-calibration of experimental variables such as stimulus duration, inter-stimulus-intervals, randomization, number of repetitions or blocks of stimuli, and control of the patient video and audio channels. StrongView streams the patient video camera, the online ECoG data, and the stimulus that the patient is currently seeing/hearing to a desktop display, which is also mirrored on a large monitor that is in the line of sight of the surgeon.

6. Attach a photodiode to the patient monitor and feed into an open channel on the ECoG amplifier. This provides a temporal sync between presentation of each stimulus and ECoG for offline analysis.

7. Use cranial navigation hardware and software (at the University of Rochester, BrainLab Inc., Munich, Germany) in all cases by the surgical team for intra-operative cranial navigation based on pre-operative MRI. This is an optical system consisting of a set of cameras that view the operating field and register the patient's head via a fixed registration star that is affixed to the operating table (see Figure 1).

1. Specifically, after the patient is set in the headholder, but before draping, use the facial physiognomy of the patient to register the patient's head to the preoperative MRI. This allows pre-operative MRI (functional and structural) to be brought into direct alignment with the patient's brain on the operating table.

2. Attach a second (much smaller) registration star to the bipolar stimulator (see Figure 1) and use to register the stimulator's length and position in the field. This enables the research team to acquire the precise location of each point of stimulation as well as the margins of the resection, relative to pre-operative MRI. As noted above, StrongView is connected with the cranial navigation system (at University of Rochester, BrainLab, connection via IGT link) to allow for real time streaming (and time stamping) of the coordinates of direct electrical stimulation mapping. StrongView is currently being developed to interface with other cranial navigation systems (e.g., Stryker).

NOTE: Aspects of StrongView that support administration and data collection during cognitive and fMRI experiments, together with a library of tests, will be available (open access) at OpenBrainProject.org. Beta versions are available prior to the full release by contacting the corresponding author. The entire StrongView suite, which includes hardware systems to integrate with 
Electrocorticography and cranial navigation software, is available to clinicians and scientists by contacting the corresponding author. These data acquisition tools will be confluent with a post-processing pipeline and open data consortium, to be launched in 2020 at OpenBrainProject.org.

\section{Representative Results}

Figure 2, Figure 3, and Figure 4 present representative results of pre-operative functional and structural mapping for three patients with tumors that were adjacent to eloquent regions of the brain. The findings shown in Figure 2, Figure 3, and Figure 4 are intended to be illustrative (rather than an exhaustive summary) of the types of maps that are generated for each patient. Details on the cases presented in Figure

2, Figure 3, and Figure 4 can be found in: Figure 2 (Chernoff, Teghipco, Garcea, Sims, Belkhir, Paul, Tivarus, Smith, Hintz, Pilcher, Mahon, in press ${ }^{51}$ ), Figure 3 (Chernoff, Sims, Smith, Pilcher and Mahon, 2019 ${ }^{52}$ ), and Figure 4 (Garcea et al., 2017 ${ }^{16}$ ). An important consequence of consecutive recruitment of glioma patients into a uniform protocol is that it makes possible group-level analyses that evaluate the effect of brain tumors on network function and organization. As an example of this type of analyses, Figure 5 presents results from a recent study ${ }^{14}$ that found that tumors in left parietal cortex modulated neural responses to 'tools' (small manipulable objects) in the temporal lobe, an instance of a more general phenomenon referred to as dynamic diaschesis ${ }^{53}$. 
A. Example setup for high through-put cognitive neuropsychological testing as

implemented by the Program for Translational Brain Mapping (Univ. of Rochester Med Ctr)

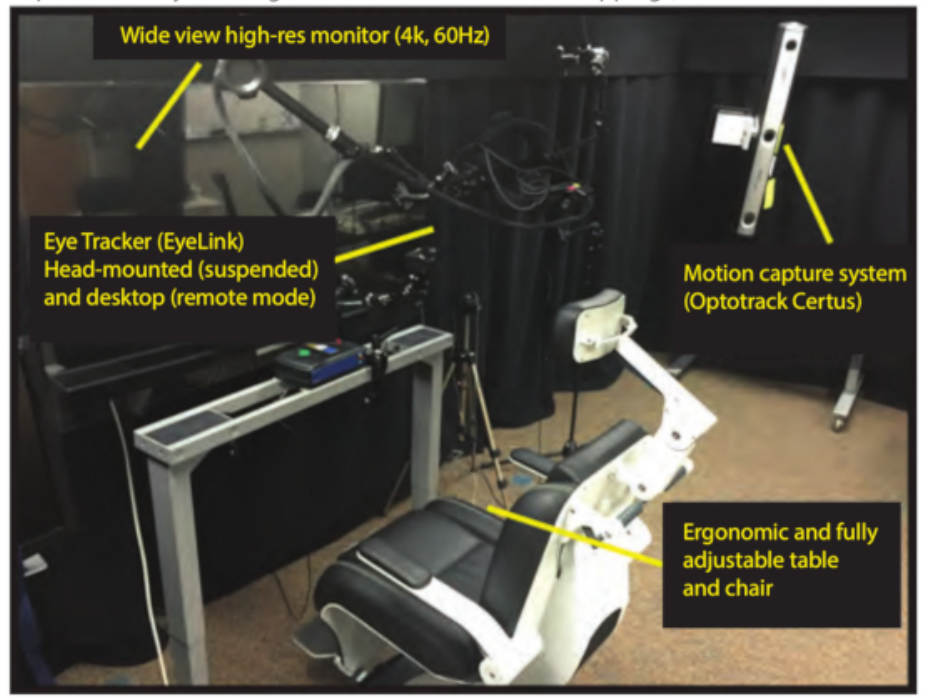

B. Equipment used during intraoperative mapping
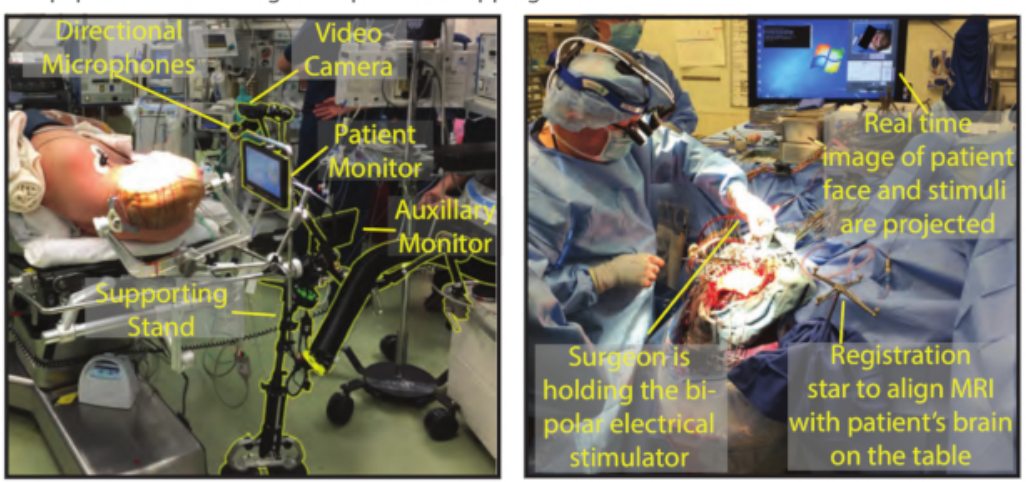

C. Bipolar Stimulator with registration star attached to record locations of intra-operative stimulation in preoperative MRI dicom space

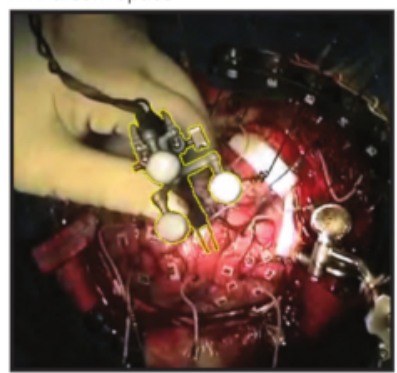

Figure 1. Overview of Equipment used for extra-operative and intra-operative cognitive testing. (A) Example setup for high throughput cognitive neuropsychological testing as implemented by the Program for Translational Brain Mapping in the Department of Neurosurgery at the University of Rochester Medical Center. Key elements for ensuring that all recruited patients are able to complete all planned tests include: $\boldsymbol{i}$ ) a place for patients to sit and complete testing that is fully adjustable to each patient's size, including a chair specifically designed to reduce fatigue, and ii) locating cognitive/behavioral testing physically adjacent to the MRI. These elements allow patients to visit the facility and complete their functional and structural MRI within the same session as core behavioral data are measured. Participants complete more trials with better performance if they are comfortable, especially for older participant populations with other comorbidities that can make sitting for prolonged periods uncomfortable. (B) Equipment used during intraoperative mapping. The image at the left shows a patient before being draped (right is after draping). Before draping, the cognitive science team sets up their equipment, including audio and video recorders of the patient, a monitor positioned in front of the patient's line of sight, and a second monitor positioned so that the person working with the patient can easily see the stimulus at which the patient is currently looking (see 'Procedure' for details). (C) Bipolar Stimulator with registration star attached to record locations of intra-operative stimulation in preoperative MRI DICOM space. Usually at the point in the surgery at which the dura has been retracted and the patient is being awoken from general anesthesia, there are a few minutes in which to register the bipolar stimulator to the field. This must be done by a team member who is scrubbed in to the case (i.e., either attending or resident surgeon or a scrub tech/nurse). It 
is accomplished by attaching a small registration star to the bipolar stimulator and following the instructions in the cranial navigation system to register a new instrument on the field. Please click here to view a larger version of this figure.

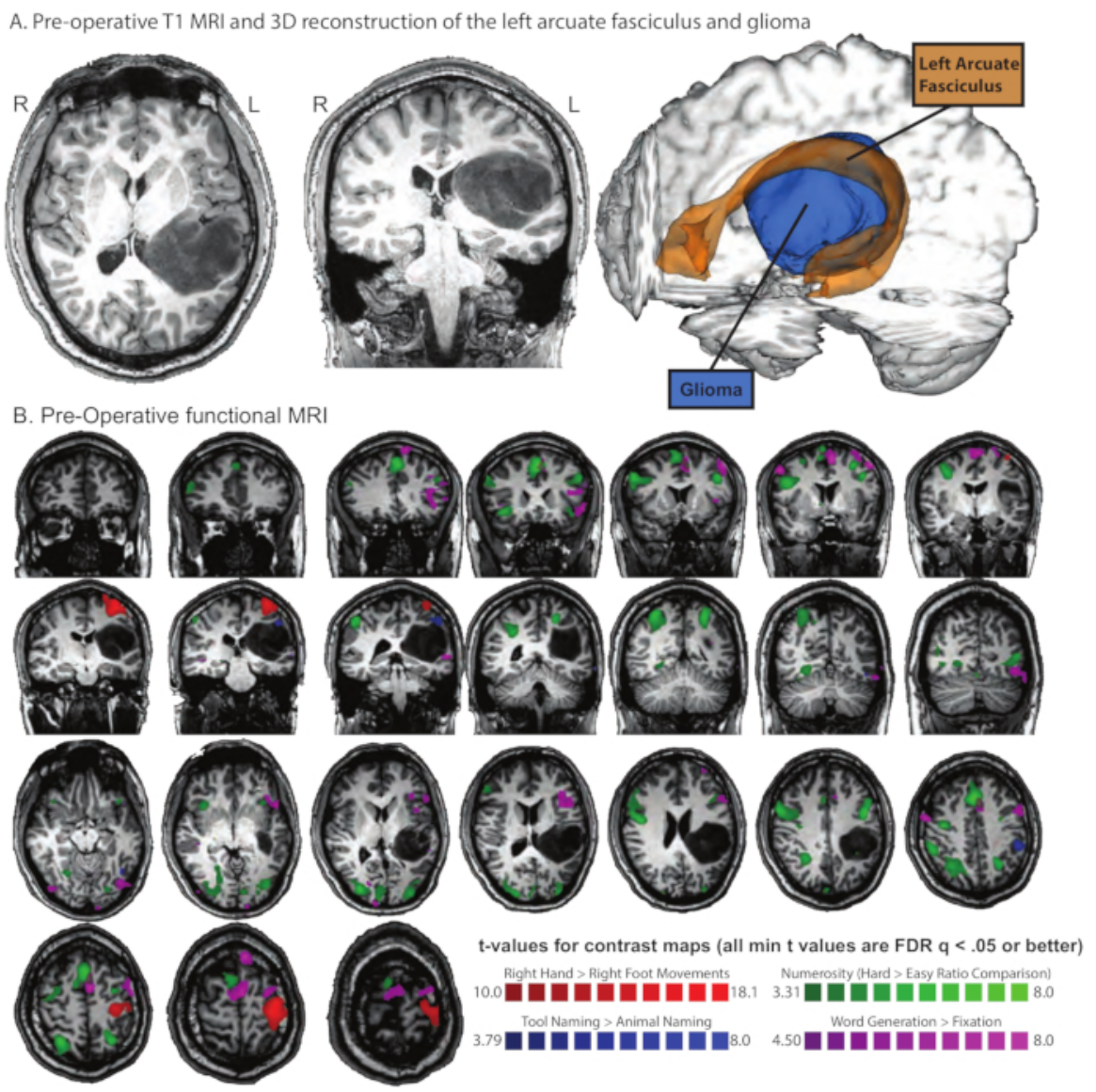

Figure 2. Pre-operative functional MRI and Diffusion Tensor Imaging (DTI) in patient AH with a left inferior parietal glioma that infiltrated the arcuate fasciculus. (A) Pre-operative T1 MRI and 3D reconstruction of the left arcuate fasciculus and glioma. The arcuate fasciculus is shown in orange at a $5 \%$ threshold with the tumor reconstructed in blue. (B) Pre-Operative functional MRI. The patient completed several sessions of functional MRI that were each designed to map a function that was anticipated to be adjacent to the area of surgical intervention. All maps are thresholded at FDR q $<.05$ or better. In blue are voxels that exhibit differential neural responses when naming tools compared to animals; in line with prior studies from our lab using the same stimuli, a robust network is identified involving premotor, parietal, and lateral and ventral temporal areas $7,8,9,10,14,15,17,18,19,20,21,22,28$. The patient was also asked to carry out a numerosity task in which he had to judge which of two clouds of dots had more dots; the two clouds of dots could either have a similar number of dots (hard comparison, ratio $=0.8$ ) or very different numbers of dots (easy comparison, ratio $=0.25$ ). In green are voxels that exhibit differential neural responses when carrying out the task over hard ratio stimuli (ratio $=.8$ ) compared to easy stimuli $\left(\right.$ ratio $=.25^{54,55}$ ). The patient was also asked to move his hands and feet (either flex/extension or rotate ${ }^{25}$ ). In red are voxels that exhibited differential neural responses to movements of the right hand compared to movements of the right foot. Finally, the patient was asked to generate as many items as he could think of in 30 seconds from various categories (e.g., 'things you do in the kitchen', 'animals', words that start with ' $F$ ', etc.). In purple are voxels that exhibited differential neural activity for overt word production compared to fixation/rest. Please click here to view a larger version of this figure. 
A. Coronal slices showing the frontal aslant tract (blue-light blue) and u-shaped fibers (red-yellow) passing anterior and medial to tumor
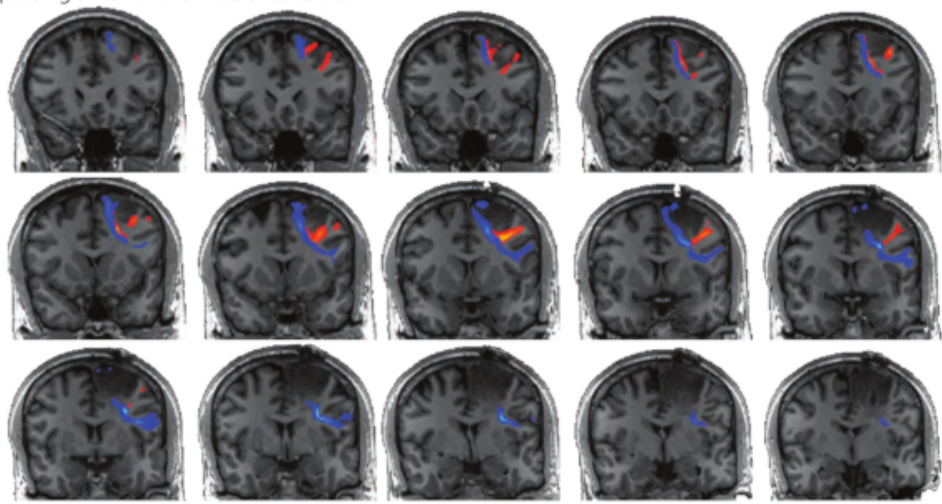

B. 3D Rendering of frontal aslant tract (blue) and tumor (red) from multiple perspectives
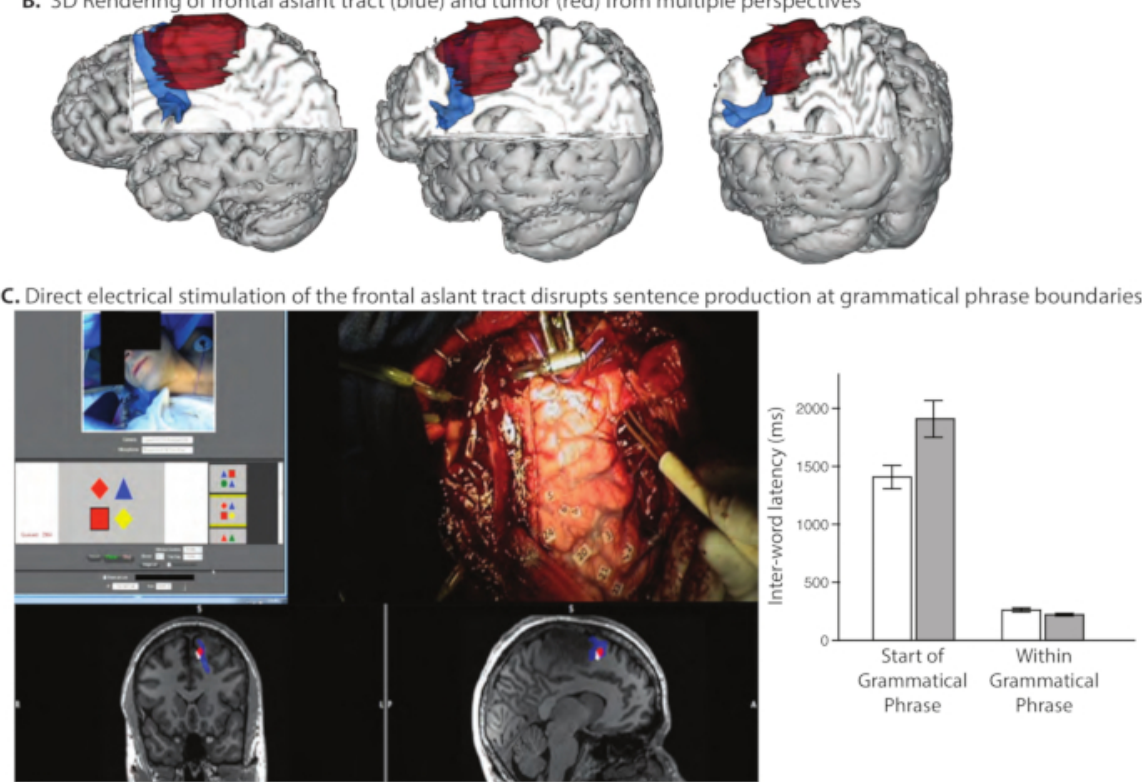

Figure 3. Pre-Operative white matter tractography of the Frontal Aslant Tract and adjacent u-shaped fibers. Prior experience in the Program for Translational Brain Mapping (Chernoff et al., $2017^{56}$ ) with brain mapping in patients with gliomas adjacent to the frontal aslant tract demonstrated that (even partial) transection of this pathway can be associated with dysfluencies in spontaneous speech, while repetition of spoken language can remain intact. That prior experience was used to inform pre-operative mapping of the frontal aslant tract in patient $\mathrm{Al}^{11}$. (A) Coronal slices showing the frontal aslant tract (blue-light blue) and u-shaped fibers (red-yellow). The frontal aslant tract passes just anterior and medial to the glioma. (B) 3D Rendering of frontal aslant tract (blue) and tumor (red) from multiple perspectives. The pre-operative anatomical studies (Panels $A$ and $B$ ) indicated that at the end of the tumor resection, it would be possible to define the anterior margin of tumor using direct electrical stimulation mapping. We thus designed a new language task based on our prior experience, specifically to test whether stimulation of the frontal aslant tract disrupted sentence production at the boundaries of grammatical phrases. (C) Direct electrical stimulation of the frontal aslant tract disrupts sentence production differentially at the boundaries of grammatical phrases. The screenshot (Panel C, left) from the video shows the patient, the stimulus with which he was presented, the surgeon's hand holding the bipolar stimulator in contact with the frontal aslant tract at the anterior margin of the tumor, and the location in coronal and sagittal slices of the current stimulation location (red dot) in relation to the frontal aslant tract (blue). The patient's task was to describe the spatial relation of the target shape in relation to the location of a reference shape (for the trial shown, the correct response would be: "The red square is below the red diamond'). We found that stimulation of the frontal aslant tract disrupted sentence production, and differentially so at the start of new grammatical phrases (Panel C, graph at right; for video of the intraoperative mapping procedure in this patient, see www.openbrainproject.org). This observation motivates a novel hypothesis about the role of the frontal aslant tract in sentence production: the Syntagmatic Constraints on Positional Elements (SCOPE) hypothesis ${ }^{11}$. Please click here to view a larger version of this figure. 

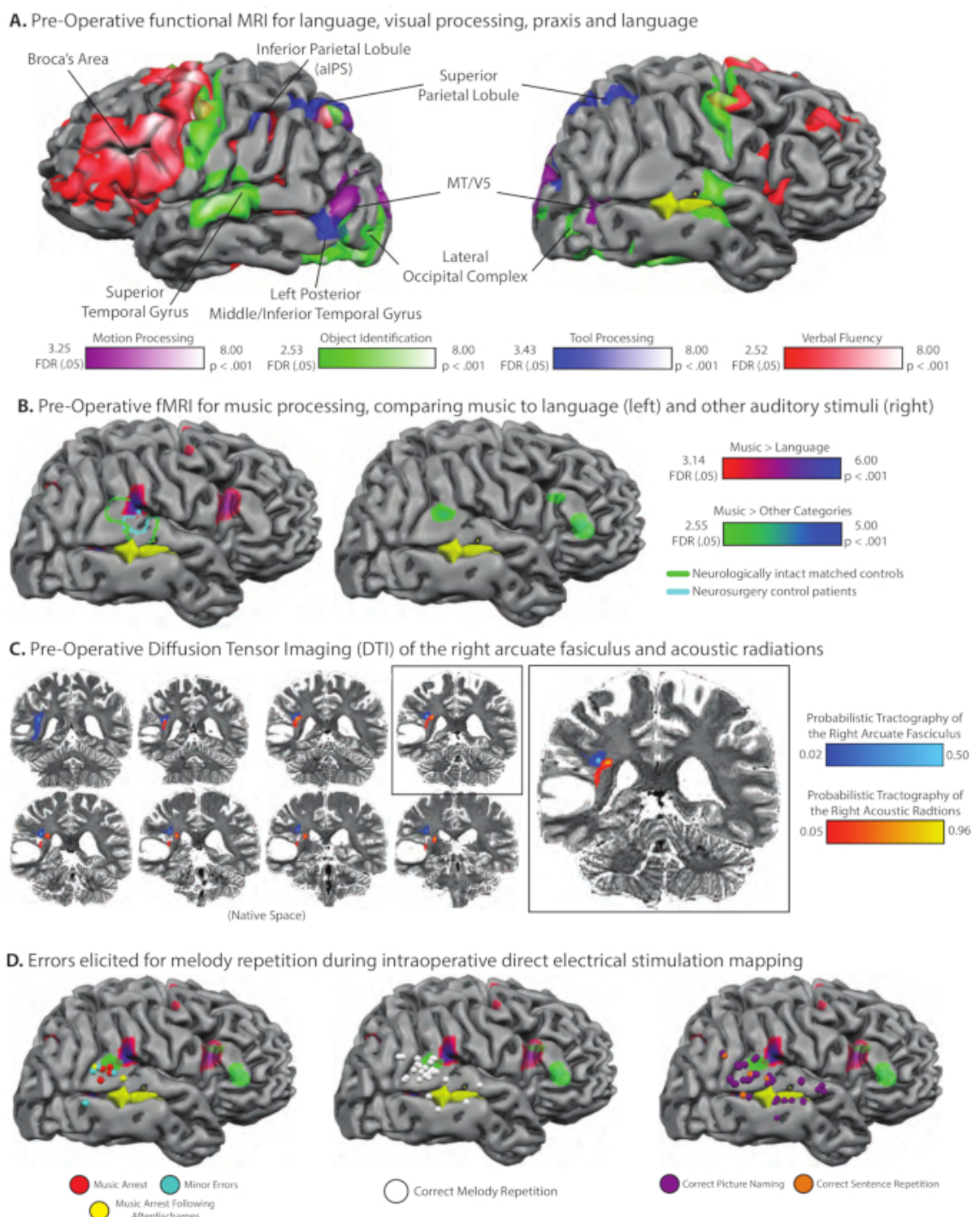

Figure 4. Pre-Operative functional and structural MRI and intraoperative direct electrical stimulation mapping in a professional musician with a glioma in the right posterior temporal lobe. (A) Pre-operative fMRI mapping of high-level visual processing, language production, and tool knowledge. The tumor, shaded yellow, was in the right temporal lobe, visible through the right superior temporal sulcus (sulci slightly expanded to facilitate visualization). Because the tumor was located close to motion processing areas in lateral temporal cortex, we localized MT/V5 by comparing neural activity when the patient attended to arrays of moving dots to neural activity elicited by stationary dots; voxels exhibiting differential neural responses for motion compared to static dots are plotted on the purple-white color scale (we are grateful to Duje Tadin for assistance with developing this functional localizer). As for all other cases studied in the Program for Translational Brain Mapping (e.g., Figure 2, Figure 3), voxels exhibiting differential neural responses for naming common pictures are compared to a baseline of viewing phase-scrambled versions of the same images; this is plotted on the green-white color scale. That contrast identified bilateral lateral occipital complex, bilateral middle/superior temporal gyrus, and motor cortex (associated with speech motor activity). Also as in Figure 2, voxels exhibiting differential neural responses when naming 'tools' were found in the left inferior parietal lobule, bilateral superior parietal/dorsal occipital cortex, and the left posterior middle/inferior temporal gyrus (blue-white color scale). Finally, and again as in Figure 2, the patient was asked to complete a verbal fluency word production task. Voxels associated with word generation compared to a resting baseline are plotted on the redwhite color scale and were found in the left inferior frontal gyrus (Broca's area), superior temporal/inferior parietal cortex, and the speech motor system. (B) The patient completed multiple functional MRI experiments pre-operatively specifically to map music processing. In one experiment, modeled after prior work from Greg Hickok's lab ${ }^{57}$, the patient heard short piano melodies and had to hum the melody back, or heard short sentences and had to repeat the sentences back. Plotted on the brain on the red-purple color scale are voxels that exhibited differential neural activity for music than for language. Four Eastman School of Music Graduate students completed the same fMRI experiment; the border of the region identified for the same functional contrast in the matched healthy controls is plotted in green outline. In addition, 10 other neurosurgery patients completed the same experiment, also in the preoperative phase of their treatment. While the proximate goal in those 10 patients was to identify language-responsive areas (thorugh the contrast of language > music), the contrast of music >language identifies a very similar region of the right superior temporal gyrus (borders of the functional region from the 10 control neurosurgery patients are drawn in light blue). (C) Preoperative probabilistic tractography over DTI data showing the right acoustic radiations and arcuate fasciculus in relation to patient AE's tumor ( $5 \%$ threshold, overlaid on native T2-weighted image). (D) During his surgery, patient AE performed the same task as during fMRI in which he had to listen to short piano melodies and hum them back, or a short sentence and repeat it back. It was found that direct electrical stimulation to the right posterior superior temporal gyrus disrupted performance in the repetition task when performed over melodies (for some trials), but 
did not affect performance (on any trials) for the same repetition task performed over sentences (see www.openbrainproject.org for videos of intraoperative music mapping). Please click here to view a larger version of this figure.

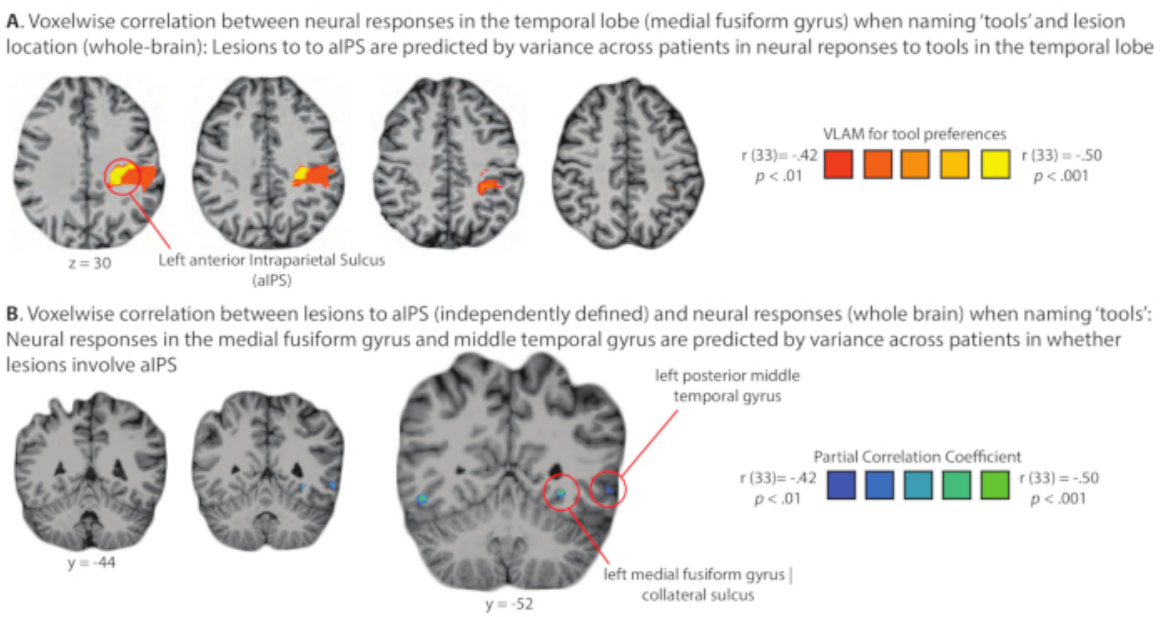

Figure 5. Demonstration of domain-specific diaschesis: Analysis of the relation of lesion location and stimulus-elicited neural activity across a group of glioma patients studied pre-operatively in the Program for Translational Brain. An important consequence of administering a common set of functional MRI and behavioral studies to all patients who go through the Program for Translational Brain Mapping at the University of Rochester Medical Center is the opportunity to carry out group-level analyses on larger sets of consecutively studied patients. As an example, Figure $\mathbf{5}$ shows the results of a test of the basic science hypothesis that neural responses to 'tools' in the temporal lobe are modulated online by inputs from parietal cortex. If that hypothesis is correct, then lesions (tumors) in parietal cortex should alter neural responses in the temporal lobe to 'tools', and variance across patients in neural activity to 'tools' in the temporal lobe should be correlated with the presence of lesions (tumors) in parietal cortex. (A) Lesions to parietal cortex are predicted at the group level (logistic regression) from variance across patients in neural responses in the medial fusiform gyrus on the ventral surface of the temporal lobe. (B) Neural responses to tools in the medial fusiform gyrus are predicted at the group level (logistic regression) from variance in whether lesion/tumor involves the anterior Intraparietal Sulcus (alPS). The findings summarized in panels A and B represent an instance of dynamic diaschesis ${ }^{53}$, in this case 'domain-specific' dynamic diaschesis, because the relation of lesion location to neural activity is modulated by the type of stimulus being processed (i.e., the relation is present for tools, and not for places, face or animals)-for full details see Garcea and colleagues ${ }^{14}$. Please click here to view a larger version of this figure.

\section{Discussion}

The knowledge gained from the experience of establishing the Program for Translational Brain Mapping at the University of Rochester can be distilled down into two core elements. First, structured channels of communication were established among cognitive scientists, neurooncologists, neuropsychologists, epileptologists, neurophysiologists, neuro-anesthesiologists, neurosurgeons and their respective supporting technicians and administrative support. This allows patients, including urgent high-grade tumor patients, to be referred for pre-operative evaluation with sufficient time to turn analyses around to surgeons prior to the procedure. The second component critical to the success of the Brain Mapping Program has been to fold in training opportunities for undergraduate students, graduate (MS, PhD) students, medical students, as well as neurosurgery, neurology and neuroradiology residents and fellows. The combination of those two elements serve to engage all clinical providers with the scientific objectives of the Brain Mapping Program, and ensures that basic science objectives are intertwined with the clinical goal of optimizing the outcome of every patient.

\section{Disclosures}

A provisional patent (U.S. Provisional Patent Number 62/917,258) was filed 11/30/18 for "StongView: An integrated hardware/software system to facilitate cognitive testing during awake brain surgery and to support real-time analyses in the service of predicting patient outcome."

\section{Acknowledgments}

This work was supported by NIH Grants R21NS076176, R01NS089069, R01EY028535, and NSF Grant BCS-1349042 to BZM, and by a University of Rochester Center for Visual Science predoctoral training fellowship (NIH training Grant 5T32EY007125-24) to FEG. We are grateful to Keith Parkins for his work on the development of StrongView, which was supported by core grant P30EY00131 to the Center for Visual Science at the University of Rochester Medical School. The Program for Translational Brain Mapping at the University of Rochester was established, in part, with support from Norman and Arlene Leenhouts, and with a grant from the Wilmot Cancer Institute to Drs. Kevin Walter and Bradford Mahon. Information about the Program for Translational Brain Mapping at the University of Rochester Medical Center can be found at: www.tbm.urmc.edu. 


\section{References}

1. Brown, T. J. et al. Association of the extent of resection with survival in glioblastoma: A systematic review and meta-analysis. JAMA Oncology. 2, 1460-1469 (2016).

2. Bloch, O.et al. Impact of extent of resection for recurrent glioblastoma on overall survival. Journal of Neurosurgery. 117, 1032 (2012).

3. McGirt, M. J.et al. Association of surgically acquired motor and language deficits on overall survival after resection of glioblastoma multiforme. Neurosurgery. 65, 463-470 (2009).

4. Rahman, M.et al. The effects of new or worsened postoperative neurological deficits on survival of patients with glioblastoma. Journal of Neurosurgery. 127, 123-131 (2017).

5. Herbet, G., Moritz-Gasser, S., Lemaitre, A. L., Almairac, F., Duffau, H. Functional compensation of the left inferior longitudinal fasciculus for picture naming. Cognitive Neuropsychology. 1-18 (2018).

6. Rofes, A.et al. Language processing from the perspective of electrical stimulation mapping. Cognitive Neuropsychology. 1-23 (2018).

7. Almeida, J., Fintzi, A. R., Mahon, B. Z. Tool manipulation knowledge is retrieved by way of the ventral visual object processing pathway. Journal of Cognitive Neuroscience. 49, 2334-2344 (2013).

8. Chen, Q., Garcea, F. E., Almeida, J., Mahon, B. Z. Connectivity-based constraints on category-specificity in the ventral object processing pathway. Neuropsychologia. 105, 184-196 (2017).

9. Chen, Q., Garcea, F. E., Jacobs, R. A., Mahon, B. Z. Abstract Representations of Object-Directed Action in the Left Inferior Parietal Lobule. Cerebral Cortex. 28, 2162-2174 (2018).

10. Chen, Q., Garcea, F. E., Mahon, B. Z. The Representation of Object-Directed Action and Function Knowledge in the Human Brain. Cerebral Cortex. 26, 1609-1618 (2016).

11. Chernoff, B., Sims, M., Smith, S., Pilcher, W., Mahon, B. Direct electrical stimulation (DES) of the left Frontal Aslant Tract disrupts sentence planning without affecting articulation. Cognitive Neuropsychology. (In Press).

12. Erdogan, G., Chen, Q., Garcea, F. E., Mahon, B. Z., Jacobs, R. A. Multisensory Part-based Representations of Objects in Human Lateral Occipital Cortex. Journal of Cognitive Neuroscience. 28, 869-881 (2016).

13. Fintzi, A. R., Mahon, B. Z. A bimodal tuning curve for spatial frequency across left and right human orbital frontal cortex during object recognition. Cerebral Cortex. 24, 1311-1318 (2014).

14. Garcea, F. E.et al. Domain-Specific Diaschisis: Lesions to Parietal Action Areas Modulate Neural Responses to Tools in the Ventral Stream. Cerebral Cortex. (2018).

15. Garcea, F. E., Chen, Q., Vargas, R., Narayan, D. A., Mahon, B. Z. Task- and domain-specific modulation of functional connectivity in the ventral and dorsal object-processing pathways. Brain Structure and Function. 223, 2589-2607 (2018).

16. Garcea, F. E.et al. Direct Electrical Stimulation in the Human Brain Disrupts Melody Processing. Current Biology. 27, 2684-2691 e2687 (2017).

17. Garcea, F. E., Kristensen, S., Almeida, J., Mahon, B. Z. Resilience to the contralateral visual field bias as a window into object representations. Journal of Cognitive Neuroscience. 81, 14-23 (2016).

18. Garcea, F. E., Mahon, B. Z. Parcellation of left parietal tool representations by functional connectivity. Neuropsychologia. 60, 131-143 (2014).

19. Kersey, A. J., Clark, T. S., Lussier, C. A., Mahon, B. Z., Cantlon, J. F. Development of Tool Representations in the Dorsal and Ventral Visual Object Processing Pathways. Cerebral Cortex. 26, 3135-3145 (2016).

20. Kristensen, S., Garcea, F. E., Mahon, B. Z., Almeida, J. Temporal Frequency Tuning Reveals Interactions between the Dorsal and Ventral Visual Streams. Journal of Cognitive Neuroscience. 28, 1295-1302 (2016).

21. Lee, D., Mahon, B. Z., Almeida, J. Action at a distance on object-related ventral temporal representations. Journal of Cognitive Neuroscience. 117, 157-167 (2019).

22. Mahon, B. Z., Kumar, N., Almeida, J. Spatial frequency tuning reveals interactions between the dorsal and ventral visual systems. Journal of Cognitive Neuroscience. 25, 862-871 (2013).

23. Paul, D. a.et al. White matter changes linked to visual recovery after nerve decompression. Science Translational Medicine. 6, 1-11 (2014).

24. Schneider, C. L.et al. Survival of retinal ganglion cells after damage to the occipital lobe in humans is activity dependent. Proceedings. Biological sciences / The Royal Society. 286, 20182733 (2019).

25. Shay, E. A., Chen, Q., Garcea, F. E., Mahon, B. Z. Decoding intransitive actions in primary motor cortex using fMRI: toward a componential theory of 'action primitives' in motor cortex. Journal of Cognitive Neuroscience. 10, 13-19 (2019).

26. Gotts, S. J. et al. Two distinct forms of functional lateralization in the human brain. Proceedings of the National Academy of Sciences of the United States of America. 110, E3435-3444 (2013).

27. Saad, Z. S. et al. Correcting brain-wide correlation differences in resting-state FMRI. Brain Connect. 3, 339-352 (2013).

28. Mahon, B. Z. et al. Action-related properties shape object representations in the ventral stream. Neuron. 55, 507-520 (2007).

29. Negri, G. A. L. et al. What is the role of motor simulation in action and object recognition? Evidence from apraxia. Cognitive Neuropsychology. 24, 795-816 (2007).

30. Stasenko, A., Garcea, F. E., Dombovy, M., Mahon, B. Z. When concepts lose their color: a case of selective loss of knowledge of object-color. Journal of Cognitive Neuroscience. 58, 217-238 (2014).

31. Stasenko, A. et al. A causal test of the motor theory of speech perception: a case of impaired speech production and spared speech perception. Cognitive Neuropsychology. 32, 38-57 (2015).

32. Garcea, F. E., Dombovy, M., Mahon, B. Z. Preserved tool knowledge in the context of impaired action knowledge: implications for models of semantic memory. Frontiers in Human Neuroscience. 7, 1-18 (2013).

33. Draper, I. T. The accessment of aphasia and related disorders. Journal of Neurology, Neurosurgery \& Psychiatry. 36, $894-895$ (1973).

34. Catani, M. et al. A novel frontal pathway underlies verbal fluency in primary progressive aphasia. Brain. 136, $2619-2628$ (2013).

35. Mesulam, M. M., Wieneke, C., Thompson, C., Rogalski, E., Weintraub, S. Quantitative classification of primary progressive aphasia at early and mild impairment stages. Brain. 135, 1537-1553 (2012).

36. Rogalski, E. et al. Progression of language decline and cortical atrophy in subtypes of primary progressive aphasia. Neurology. $\mathbf{7 6}$, 1804-1810 (2011). 
37. Snodgrass, J. G., Vanderwart, M. A standardized set of 260 pictures: norms for name agreement, image agreement, familiarity, and visual complexity. Journal of Experimental Psychology: Human Learning and Memory. 6, 174-215 (1980).

38. Hamberger, M. J., Seidel, W. T. Auditory and visual naming tests: Normative and patient data for accuracy, response time, and tip-of-thetongue. Journal of the International Neuropsychological Society. 9, 479-489 (2003).

39. Riddoch, M., Humphreys, J., Glyn, W. Birmingham object recognition battery. Psychology Press (1993).

40. Kay, J., Lesser, R., Coltheart, M. Psycholinguistic assessments of language processing in aphasia (PALPA). (1992).

41. Farnsworth, D. The Farnsworth-Munsell 100-hue and dichotomous tests for color vision. Journal of the Optical Society of America. 33, 568-578 (1943).

42. Duchaine, B., Nakayama, K. The cambridge face memory test: results for neurologically intact individuals and an investigation of its validity using inverted face stimuli and prosopagnosic participants. Neuropsychologia. 44, 576-585 (2006).

43. Gorno-Tempini, M. L. et al. Cognition and anatomy in three variants of primary progressive aphasia. Annals of Neurology. 55, 335-346 (2004).

44. Weiss, L., Saklofske, D., Coalson, D., Raiford, S. WAIS-IV clinical use and interpretation: scientist-practitioner perspectives. Practical Resources for the Mental Health Professional. (2010).

45. Canizares, S. et al. Reliability and clinical usefulness of the short-forms of the Wechsler memory scale (revised) in patients with epilepsy. Epilepsy Research. 41, 97-106 (2000).

46. Wechsler, D. The measurement and appraisal of adult intelligence, 4th ed. Williams \& Wilkins (1958).

47. Caramazza, A. The logic of neuropsychological research and the problem of patient classification in aphasia. Brain and Language. 21, 9-20 (1984).

48. Sanai, N., Mirzadeh, Z., Berger, M. S. Functional outcome after language mapping for glioma resection. New England Journal of Medicine. 358, 18-27 (2008).

49. Ojemann, G. Individual variability in cortical localization of language. Journal of Neurosurgery. 50, 164-169 (1979).

50. Rofes, A., de Aguiar, V., Miceli, G. A minimal standardization setting for language mapping tests: an Italian example. Neurological Sciences. 36, 1113-1119 (2015)

51. Chernoff, B.L., Teghipco, A., Garcea, F.E., Sims, M.H., Belkhir, R., Paul, D.A., Tivarus, M.E., Smith, S.O., Hintz, E., Pilcher, W.H., Mahon, B.Z. Reorganized language network connectivity after left arcuate fasciculus resection: A case study. Cortex. (in press).

52. Chernoff, B.L., Sims, M.H., Smith, S.O., Pilcher W.H., and Mahon B.Z. Direct electrical stimulation of the left frontal aslant tract disrupts sentence planning without affecting articulation. Cognitive Neuropsychology. 36,(2019).

53. Price, C. J., Warburton, E. A., Moore, C. J., Frackowiak, R. S., Friston, K. J. Dynamic diaschisis: anatomically remote and context-sensitive human brain lesions. Journal of Cognitive Neuroscience. 13, 419-429 (2001)

54. Kersey, A. J., Cantlon, J. F. Neural Tuning to Numerosity Relates to Perceptual Tuning in 3-6-Year-Old Children. Journal of Neuroscience. 37 512-522 (2017).

55. Kersey, A. J., Cantlon, J. F. Primitive Concepts of Number and the Developing Human Brain. Language Learning and Development. 13, 191-214 (2017).

56. Chernoff, B.L., Teghipco, A., Garcea, F.E., Sims, M. H., Paul, D.A., Tivarus, M.E., Smith, S.O., Pilcher, W.H., \& Mahon, B.Z. A Role for the Frontal Aslant Tract in Speech Planning: A Neurosurgical Case Study. Journal of Cognitive Neuroscience. 30 (5), 752-769. (2018).

57. Hickok, G., Buchsbaum, B., Humphries, C., Muftuler, T. Auditory-motor interaction revealed by fMRI: speech, music, and working memory in area Spt. Journal of Cognitive Neuroscience. 15, 673-682 (2003). 